\title{
ON THE COHOMOLOGY RING OF HYPERPLANE COMPLEMENTS
}

\author{
RICHARD JOZSA AND JOHN RICE
}

(Communicated by Louis J. Ratliff, Jr.)

\begin{abstract}
Using only the long exact sequence of local cohomology, we give a brief derivation of Orlik and Solomon's presentation for the cohomology ring of a complement of hyperplanes in a vector space.
\end{abstract}

\section{INTRODUCTION}

Let $V$ be a complex vector space and $\alpha_{1}, \alpha_{2}, \ldots, \alpha_{m}$ a family of distinct linear functions on $V$. Let $V_{1}, V_{2}, \ldots, V_{m}$ denote the corresponding hyperplanes, i.e. their kernels, and let $X$ denote their complement in $V$, that is $X=V \backslash \bigcup_{i=1}^{m} V_{i}$. Brieskorn [2] proved that the cohomology ring of $X$ is generated by $m$ anticommuting elements, one for each hyperplane, while Orlik and Solomon [5] described the relations between them. In de Rham cohomology one can simply say that $H^{*}(X)$ is generated by (the cohomology classes of) the closed forms $d \alpha_{i} / \alpha_{i}$ for $i=1, \ldots, m$, subject to the relations

$$
\sum_{j=1}^{k}(-1)^{j} \frac{d \alpha_{i_{1}}}{\alpha_{i_{1}}} \wedge \frac{d \alpha_{i_{2}}}{\alpha_{i_{2}}} \wedge \cdots\left[\frac{d \alpha_{i_{j}}}{\alpha_{i_{j}}}\right] \cdots \frac{d \alpha_{i_{k}}}{\alpha_{i_{k}}}=0
$$

for each linearly dependent set $\alpha_{i_{1}}, \ldots, \alpha_{i_{k}}$. The bracket $[\cdots]$ in the expression indicates that the enclosed term is omitted.

It is actually very easy to see that such relations must be satisfied if one considers interior product $\Upsilon \neg \omega$ of differential forms against the Euler vector field $\Upsilon$. The Euler vector field is the infinitesimal generator of the dilation group on $V$. In any linear coordinate system $x_{1}, \ldots, x_{n}$ on $V$ it is given explicitly by

$$
\Upsilon=\sum_{i=1}^{n} x_{i} \frac{\partial}{\partial x_{i}}
$$

and it is clear that $\Upsilon \neg d \alpha / \alpha=1$ for any linear function $\alpha$. Since $\Upsilon \neg$ is a derivation of degree -1 on the algebra of differential forms it follows that, for

Received by the editors May 8, 1990.

1980 Mathematics Subject Classification (1985 Revision). Primary 32C40. 
any set of linear functions $\alpha_{i_{1}}, \ldots, \alpha_{i_{k}}$, if $\omega$ is given by

$$
\omega=\frac{d \alpha_{i_{1}}}{\alpha_{i_{1}}} \wedge \frac{d \alpha_{i_{2}}}{\alpha_{i_{2}}} \wedge \cdots \wedge \frac{d \alpha_{i_{k}}}{\alpha_{i_{k}}}
$$

then $\Upsilon \neg \omega$ gives the left-hand side of (1.1). Now $\omega$ is zero precisely when $\alpha_{i_{1}}, \ldots, \alpha_{i_{k}}$ are dependent, and in this event $\Upsilon \neg \omega$ must be zero also.

In this paper we present a very simple inductive argument, involving only the long exact sequence for local cohomology and a primitive form of the Thom isomorphism, to show that $H^{\circ}(X)$ is generated by the cohomology classes of $d \alpha_{i} / \alpha_{i}$ for $i=1, \ldots, m$ and that there are no further relations other than those derived from the ones described above. In some sense this is a simplification of the work of Orlik, Solomon, and Terao $[4,5,6]$ and in particular it involves no reference to any of the lattice theoretic constructs upon which their arguments depend. We also give a new and elementary derivation of the direct sum decomposition $[2,5,6]$ for $H^{*}(X)$.

In the hope of making the work accessible to a wider audience we treat local cohomology as relative de Rham cohomology. However the results we use and the arguments we make are independent of this de Rham interpretation, and they apply for cohomology over any ring. We elaborate on this point at the end of the paper, noting in particular that as a corollary $H^{\circ}(X, \mathbb{Z})$ is torsion free.

\section{LOCAL COHOMOLOGY}

We begin by recalling some elementary facts about local cohomology $[1,3]$. Let $M$ be a submanifold of the manifold $X$, and let $\Omega^{\circ}(X)$ and $\Omega^{\circ}(X \backslash M)$ denote the de Rham complexes of $X$ and $X \backslash M$. For the constant sheaf $\mathbb{R}$ local cohomology may be represented as the cohomology of the relative de Rham complex [1] of $(X, X \backslash M)$, whose elements in degree $k$ consist of pairs $(\omega, \psi)$ with $\omega \in \Omega^{k}(X), \psi \in \Omega^{k-1}(X \backslash M)$ and whose differential $D$ is given by

$$
D(\omega, \psi)=\left(d \omega, j^{*} \omega-d \psi\right)
$$

where $X \backslash M \stackrel{j}{\longrightarrow} X$ is the inclusion map. We denote it $\Omega^{\circ}(X, X \backslash M)$. The maps $\alpha$ and $\beta$ defined by

$$
\alpha(\omega, \psi)=\omega, \quad \beta(\psi)=(0, \psi)
$$

give a short exact sequence of complexes

$$
0 \rightarrow \Omega^{\circ}(X \backslash M) \stackrel{\beta}{\longrightarrow} \Omega^{\bullet}(X, X \backslash M) \stackrel{\alpha}{\longrightarrow} \Omega^{\bullet}(X) \rightarrow 0
$$

which results in the long exact sequence of local cohomology

$$
\cdots \rightarrow H^{k}(X) \rightarrow H^{k}(X \backslash M) \rightarrow H_{M}^{k+1}(X) \rightarrow \cdots .
$$

If $U$ is a tubular neighbourhood of $M$ in $X$ then restriction from $X$ to $U$ defines a morphism from the exact sequence $(2.1)$ for $X$ to that for $U$, which 
induces a commuting diagram

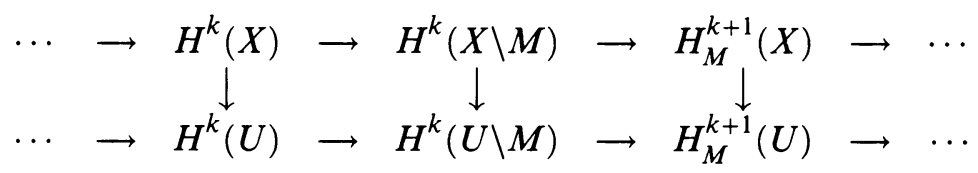

The map $H_{M}^{\cdot}(X) \rightarrow H_{M}^{*}(U)$ in the centre of the diagram is called the excision map, and the Excision Theorem states that it is an isomorphism.

We shall be concerned only with the case where $M$ is the zero set of a function $X \stackrel{f}{\longrightarrow} \mathbb{C}$ with $0 \in \mathbb{C}$ a regular value. By the submersion theorem there is a tubular neighbourhood $U \stackrel{\pi}{\longrightarrow} M$ such that $U \stackrel{\pi \times f}{\longrightarrow} M \times \mathbb{C}$ is a diffeomorphism. Under this diffeomorphism $U \backslash M$ corresponds to $M \times(\mathbb{C} \backslash 0)$. The Künneth Theorem states that the map $(\omega, \alpha) \rightarrow \pi^{*} \omega \wedge f^{*} \alpha$ induces an isomorphism $H^{*}(M) \otimes H^{*}\left(\mathbb{C}^{*}\right) \rightarrow H^{*}(U \backslash M)$ where $\mathbb{C}^{*}$ denotes $\mathbb{C} \backslash 0$. Now $H^{*}\left(\mathbb{C}^{*}\right)$ is spanned by $1 \in H^{0}\left(\mathbb{C}^{*}\right)$ and $d z / z \in H^{1}\left(\mathbb{C}^{*}\right)$ where $z$ is the natural coordinate on $\mathbb{C}$, and $f^{*}(d z / z)=d f / f$. Hence $H^{*}(U \backslash M)$ is spanned by the classes $\pi^{*} \omega$ and $\pi^{*} \omega \wedge d f / f$ where $\omega$ ranges over representatives of the cohomology classes of $H^{*}(M)$. In particular, the classes of the form $\pi^{*} \omega$ are the restrictions of such classes from $H^{*}(U)$, and by the Poincaré Lemma these constitute the whole of the cohomology of $H^{*}(U)$. The restriction map $H^{*}(U) \stackrel{j^{*}}{\longrightarrow} H^{*}(U \backslash M)$ is therefore injective.

Now consider the long exact sequence

$$
\cdots H^{k}(U) \stackrel{j^{*}}{\longrightarrow} H^{k}(U \backslash M) \stackrel{\beta}{\longrightarrow} H_{M}^{k+1}(U) \stackrel{\gamma}{\longrightarrow} H^{k+1}(U) \stackrel{j^{*}}{\longrightarrow} H^{k+1}(U \backslash M) \cdots
$$

Because $j^{*}$ is an injection $\beta$ is a surjection and we have a short exact sequence

$$
0 \rightarrow H^{k}(U) \stackrel{j^{*}}{\longrightarrow} H^{k}(U \backslash M) \stackrel{\beta}{\longrightarrow} H_{M}^{k+1}(U) \rightarrow 0 .
$$

The map $\beta$ is induced by $\psi \rightarrow(0, \psi)$ and its kernel consists of the classes of the form $\pi^{*} \omega$. Thus $H_{M}^{k+1}(U)$ is spanned by the classes $\left(0, \pi^{*} \omega \wedge d f / f\right)$ where $\omega$ ranges over representatives of $H^{k-1}(M)$. This provides an explicit Thom isomorphism $H^{k-1}(M) \rightarrow H_{M}^{k+1}(U)$ and shows that $(0, d f / f)$ represents the Thom class in $H_{M}^{2}(U)$.

\section{THE COHOMOLOGY OF A HYPERPLANE COMPLEMENT}

Let $X$ be a complement of hyperplanes $V_{1}, \ldots, V_{m}$ as described in the introduction. We first prove Brieskorn's result

Theorem 1. $H^{*}(X)$ is generated by the cohomology classes of the differential forms $d \alpha_{i} / \alpha_{i}, i=1, \ldots, m$.

Proof. We argue by induction on the number of hyperplanes. Assume therefore that the result is true for the complement of any $m$ hyperplanes in any vector space. Let $X$ be as described, and let $V_{0}$ be a further hyperplane which is the 
kernel of the linear function $\alpha_{0}$. We let $M=X \cap V_{0}$ so that $X \backslash M$ is the complement of all the hyperplanes $V_{0}, V_{1}, \ldots, V_{m}$. Consider the long exact sequence

$$
\cdots H^{k-1}(X \backslash M) \stackrel{\beta}{\longrightarrow} H_{M}^{k}(X) \stackrel{\gamma}{\longrightarrow} H^{k}(X) \stackrel{j^{*}}{\longrightarrow} H^{k}(X \backslash M) \stackrel{\beta}{\longrightarrow} H_{M}^{k+1}(X) \cdots .
$$

We show that $\beta$ is surjective.

Since $M$ is the zero set in $X$ of $\alpha_{0}$ there is, as discussed in the previous section, a tubular neighbourhood $U$ such that $H^{k}(U \backslash M) \stackrel{\beta^{\prime}}{\longrightarrow} H_{M}^{k+1}(U)$ is surjective. We also have the excision diagram

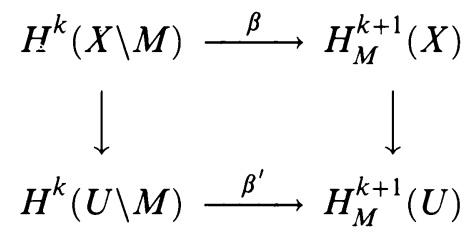

where the excision map, the vertical map on the right, is an isomorphism. The cohomology classes which span $H_{M}^{k+1}(U)$ are the images under $\beta^{\prime}$ of $\pi^{*} \omega \wedge$ $d \alpha_{0} / \alpha_{0}$, where $\pi$ can be taken as the restriction to $X$ of a linear projection $V \rightarrow V_{0}$, and $\omega$ runs through representatives of the cohomology classes of $M$.

Now $M$ is itself the complement in $V_{0}$ of the hyperplanes defined by the restrictions of $\alpha_{1}, \ldots, \alpha_{m}$ to $V_{0}$. By our inductive hypothesis, $H^{*}(M)$ is generated by the cohomology classes of $i^{*} d \alpha_{1} / \alpha_{1}, \ldots, i^{*} d \alpha_{m} / \alpha_{m}$ where $M \stackrel{i}{\longrightarrow} U$ is the inclusion map. By the Poincare Lemma $\pi^{*}$ is an inverse to $i^{*}$ in cohomology, so we can conclude that the cohomology classes which span $H_{M}^{k+1}(U)$ are the images under $\beta^{\prime}$ of $\nu \wedge d \alpha_{0} / \alpha_{0}$ where $\nu$ ranges over differential forms on $U$ generated from $d \alpha_{1} / \alpha_{1}, \ldots, d \alpha_{m} / \alpha_{m}$. Taking these same forms on $X$ itself, the excision diagram shows that their images under $\beta$ spans $H_{M}^{k+1}(X)$. Thus $\beta$ is surjective.

Because $\beta$ is surjective $j^{*}$ is injective, and we obtain short exact sequences

$$
0 \rightarrow H^{k}(X) \stackrel{j^{*}}{\longrightarrow} H^{k}(X \backslash M) \stackrel{\beta}{\longrightarrow} H_{M}^{k+1}(X) \rightarrow 0 .
$$

$H^{k}(X \backslash M)$ is therefore generated by the images of a set of generators for $H^{k}(X)$ along with a set of elements whose images under $\beta$ generate $H_{M}^{k+1}(X)$. By our inductive hypothesis the former may be chosen as products of $d \alpha_{1} / \alpha_{1}, \ldots$, $d \alpha_{m} / \alpha_{m}$, while our discussion above shows that the latter can be chosen as products of these forms along with $d \alpha_{0} / \alpha_{0}$. In other words, $d \alpha_{0} / \alpha_{0}, \ldots, d \alpha_{m} / \alpha_{m}$ generate $H^{*}(X \backslash M)$. This concludes the inductive step of our argument and, since the theorem is trivial for the case $m=0$, completes the proof.

We now turn to the description of the relations between the generators $d \alpha_{1} / \alpha_{1}, \ldots, d \alpha_{m} / \alpha_{m}$ of $H^{*}(X)$. For this purpose let us consider the map $V \stackrel{\alpha}{\longrightarrow} \mathbb{C}^{m}$ whose component functions are $\left(\alpha_{1}, \ldots, \alpha_{m}\right)$. If $z_{i}$ denotes the 
$i$ th component function on $\mathbb{C}^{m}$ then $\alpha_{i}=z_{i} \circ \alpha$ and $d \alpha_{i} / \alpha_{i}=\alpha^{*}\left(d z_{i} / z_{i}\right)$. Restricting $\alpha$ to $X$ gives a map $X \stackrel{\alpha}{\longrightarrow} \mathbb{C}^{* m}$ and our theorem above asserts that the induced map $H^{*}\left(\mathbb{C}^{* m}\right) \stackrel{\alpha^{*}}{\longrightarrow} H^{*}(X)$ is a surjection. By the Künneth formula $H^{*}\left(\mathbb{C}^{* m}\right)$ is an exterior algebra generated by $d z_{1} / z_{1}, \ldots, d z_{m} / z_{m}$, i.e. there are no relations. If $\Upsilon$ is the Euler operator $\sum_{i=1}^{m} z_{i} \partial / \partial z_{i}$ then we claim [4-6].

Theorem 2. The kernel of $H^{*}\left(\mathbb{C}^{* m}\right) \stackrel{\alpha^{*}}{\longrightarrow} H^{*}(X)$ is the ideal $I_{X}$ generated by the differential forms $\Upsilon \neg\left(d z_{i_{1}} / z_{i_{1}} \wedge \cdots \wedge d z_{i_{k}} / z_{i_{k}}\right)$ for which $\alpha_{i_{1}}, \ldots, \alpha_{i_{k}}$ are linearly dependent.

Proof. Since $\alpha$ is linear $\alpha^{*}$ intertwines the action of the Euler operators on $V$ and $\mathbb{C}^{* m}$, which we denote by the same symbol $\Upsilon$. Therefore

$$
\alpha^{*}\left(\Upsilon \neg\left(\frac{d z_{i_{1}}}{z_{i_{1}}} \wedge \cdots \wedge \frac{d z_{i_{k}}}{z_{i_{k}}}\right)\right)=\Upsilon \neg \alpha^{*}\left(\frac{d z_{i_{1}}}{z_{i_{1}}} \wedge \cdots \wedge \frac{d z_{i_{k}}}{z_{i_{k}}}\right)=\Upsilon \neg\left(\frac{d \alpha_{i_{1}}}{\alpha_{i_{1}}} \wedge \cdots \wedge \frac{d \alpha_{i_{k}}}{\alpha_{i_{k}}}\right) .
$$

If $\alpha_{i_{1}}, \ldots, \alpha_{i_{k}}$ are dependent then this last expression is zero. Hence the ideal $I_{X}$ lies in the kernel of $\alpha^{*}$.

To prove the converse we argue by induction as in the proof of Theorem 1 . Let $X, M$, and $\alpha_{0}, \ldots, \alpha_{m}$ be as described there and $\alpha$ be the map $V \rightarrow$ $\mathbb{C}^{m+1}$ with components $\alpha_{0}, \ldots, \alpha_{m}$. If we regard $\mathbb{C}^{m+1}$ as $\mathbb{C} \times \mathbb{C}^{m}$ then $X$ is the inverse image under $\alpha$ of $\mathbb{C} \times \mathbb{C}^{* m}, M$ is the inverse image of $0 \times \mathbb{C}^{* m}$, and $X \backslash M$ the inverse image of $\mathbb{C} \times \mathbb{C}^{* m} \backslash 0 \times \mathbb{C}^{* m}=\mathbb{C}^{* m+1}$. From the local cohomology long exact sequence we obtain the diagram of maps

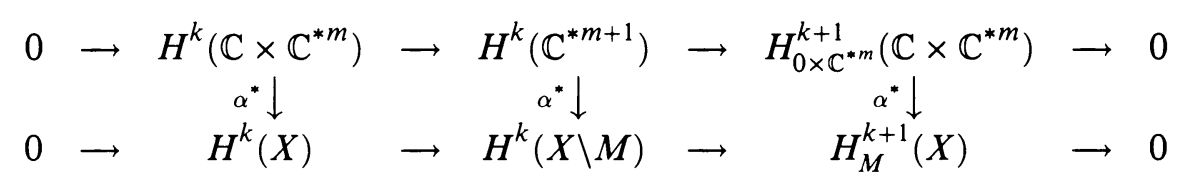

The Poincaré Lemma identifies $H^{k}\left(\mathbb{C} \times \mathbb{C}^{* m}\right)$ with $H^{k}\left(\mathbb{C}^{* m}\right)$ in such a way that the leftmost map $\alpha^{*}$ coincides with pull-back under $\alpha^{\prime}=\left(\alpha_{1}, \ldots, \alpha_{m}\right)$, and by our inductive hypothesis its kernel is $I_{X}$. Under the Thom isomorphism, as we discussed at the end of $\S 2$, the map on the right corresponds to $H^{k-1}\left(\mathbb{C}^{* m}\right) \stackrel{\alpha^{\prime *}}{\longrightarrow} H^{k-1}(M)$ where $\alpha^{\prime}$ is restricted to $M$. By our inductive hypothesis its kernel $I_{M}$ is generated by the forms $\Upsilon \neg\left(\frac{d z_{i_{1}}}{z_{i_{1}}} \wedge \cdots \wedge \frac{d z_{i_{k}}}{z_{i_{k}}}\right)$ for which $\alpha_{i_{1}}, \ldots, \alpha_{i_{k}}$ are linearly dependent when restricted to $V_{0}$. This is the same as to say that $\alpha_{0}, \alpha_{i_{1}}, \ldots, \alpha_{i_{k}}$ are linearly dependent. We therefore arrive at the 
diagram

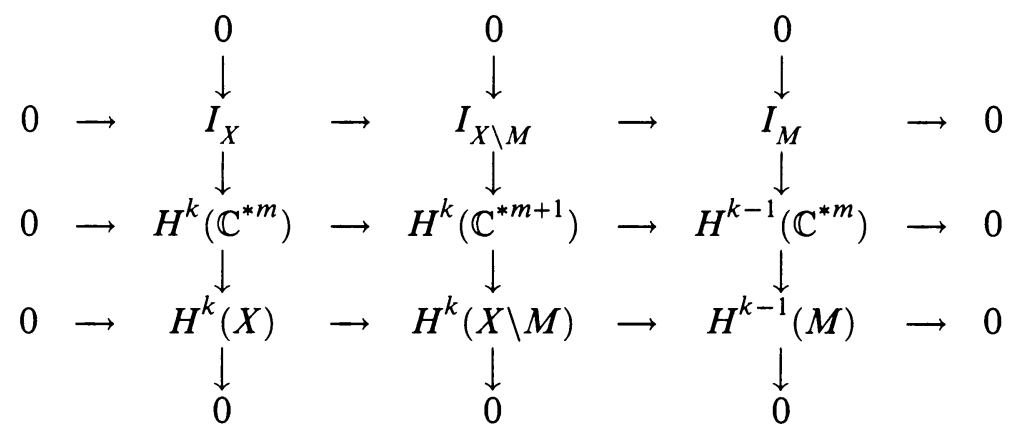

We know that the left- and right-hand columns of this diagram are exact and that the middle column is exact except at the centre. Our objective is to prove its exactness there. We also know that the bottom two rows are exact. An elementary diagram chase shows that if $I_{X \backslash M} \rightarrow I_{M}$ is surjective then the middle column is exact at the centre. Indeed, if $\nu \in H^{k}\left(\mathbb{C}^{* m+1}\right)$ maps downwards to zero then its image in $H^{k-1}\left(\mathbb{C}^{* m}\right)$ does also, and must therefore arise from an element of $I_{M}$. Assuming the surjectivity of $I_{X \backslash M} \rightarrow I_{M}$ this element is the image of some $\nu^{\prime} \in I_{X \backslash M}$ and then, using the same notation for its image in $H^{k}\left(\mathbb{C}^{* m+1}\right)$, it is clear that $\nu-\nu^{\prime}$ maps to zero in $H^{k-1}\left(\mathbb{C}^{* m}\right)$. By exactness this difference is the image of an element of $H^{k}\left(\mathbb{C}^{* m}\right)$ which maps downwards to zero, and so arises from an element of $I_{X}$. It follows that $\nu-\nu^{\prime}$ is equal to the image of this element via $I_{X \backslash M}$ and so $\nu$ itself lies in the image of $I_{X \backslash M}$.

To prove the surjectivity of $I_{X \backslash M} \rightarrow I_{M}$ we observe two consequences of the formula

$\Upsilon \neg\left(\frac{d z_{i}}{z_{i}} \wedge\left(\frac{d z_{i_{1}}}{z_{i_{1}}} \wedge \cdots \frac{d z_{i_{k}}}{z_{i_{k}}}\right)\right)=\frac{d z_{i_{1}}}{z_{i_{1}}} \wedge \cdots \frac{d z_{i_{k}}}{z_{i_{k}}}-\left(\frac{d z_{i}}{z_{i}}\right) \wedge \Upsilon \neg\left(\frac{d z_{i_{1}}}{z_{i_{1}}} \wedge \cdots \wedge \frac{d z_{i_{k}}}{z_{i_{k}}}\right)$. If $i=i_{1}$ then the left-hand side is zero, and hence $d z_{i_{1}} / z_{i_{1}} \wedge \cdots d z_{i_{k}} / z_{i_{k}}$ lies in $I_{X}$ if $\Upsilon \neg\left(d z_{i_{1}} / z_{i_{1}} \wedge \cdots d z_{i_{k}} / z_{i_{k}}\right)$ does, i.e., if $\alpha_{1}, \ldots, a_{k}$ are dependent. Secondly, we see that $I_{X}$ is actually spanned by the elements $d z_{i_{i}} / z_{i_{1}} \wedge \cdots d z_{i_{k}} / z_{i_{k}}$ and $\Upsilon \neg\left(d z_{i_{1}} / z_{i_{1}} \wedge \cdots d z_{i_{k}} / z_{i_{k}}\right)$ for which $\alpha_{i_{1}}, \ldots, \alpha_{i_{k}}$ are dependent. For even if $i$ is not one of $i_{1}, \ldots, i_{k}$, the set $\alpha_{i}, \alpha_{i_{1}}, \ldots, \alpha_{i_{k}}$ is dependent and the formula shows that $\left(d z_{i} / z_{i}\right) \wedge \Upsilon \neg\left(d z_{i_{1}} / z_{i_{1}} \wedge \cdots \wedge d z_{i_{k}} / z_{i_{k}}\right)$ lies in the span of these elements. By induction, the whole of $I_{X}$ lies in this span.

The map $I_{X \backslash M} \rightarrow I_{M}$ sends $d z_{i_{1}} / z_{i_{1}} \wedge \cdots \wedge d z_{i_{m}} / z_{i_{m}}$ to

$$
(-1)^{j} \frac{d z_{i_{1}}}{z_{i_{1}}} \wedge \cdots\left[\frac{d z_{i_{j}}}{z_{i_{j}}}\right] \cdots \wedge \frac{d z_{i_{m}}}{z_{i_{m}}}
$$

if $i_{j}=0$ and to 0 if $i_{j} \neq 0$ for any $j=1, \ldots, k$. The kernel $I_{M}$ is spanned by the elements $d z_{i_{1}} / z_{i_{1}} \wedge \cdots d z_{i_{k}} / z_{i_{k}}$ and $\Upsilon \neg\left(d z_{i_{1}} / z_{i_{1}} \wedge \cdots d z_{i_{k}} / z_{i_{k}}\right)$ 
for which the restrictions of $\alpha_{i_{1}}, \ldots, \alpha_{i_{k}}$ to the kernel of $\alpha_{0}$ are dependent. But this is equivalent to the dependence of $\alpha_{0}, \alpha_{i_{1}}, \ldots, \alpha_{i_{k}}$. Therefore $d z_{0} / z_{0} \wedge$ $\cdots d z_{i_{k}} / z_{i_{k}}$ and $\Upsilon \neg\left(d z_{0} / z_{0} \wedge \cdots d z_{i_{k}} / z_{i_{k}}\right)$ lie in $I_{X \backslash M}$ and they have images $d z_{i_{1}} / z_{i_{1}} \wedge \cdots d z_{i_{k}} / z_{i_{k}}$ and $\Upsilon \neg\left(d z_{i_{1}} / z_{i_{1}} \wedge \cdots d z_{i_{k}} / z_{i_{k}}\right)$.

This proves that $I_{X \backslash M} \rightarrow I_{M}$ is surjective and completes the inductive step of the argument. The theorem is clearly true for the case of a single hyperplane $\alpha$, for if $\alpha \neq 0$ then $I_{X}=0$ and if $\alpha=0$ then $I_{X}$ is spanned by $d \alpha / \alpha$. This finishes the proof of the theorem.

\section{THE DIRECT SUM DECOMPOSITION}

Let $W_{1}, \ldots, W_{N}$ be a complete list of codimension $r$ subspaces of $V$ which arise as the intersections of the given hyperplanes $V_{1}, \ldots, V_{m}$. For each $i=$ $1, \ldots, N$ let $X_{r_{i}}$ be the complement in $V$ of all those of the given hyperplanes containing $W_{i}$ :

$$
X_{r_{i}}=V \backslash \bigcup_{W_{i} \subseteq V_{j}} V_{j} \quad i=1, \ldots, N .
$$

(Note that for each $W_{i}$ there must be at least $r$ such hyperplanes.)

According to Theorem 1, a general element of $H^{r}(X)$ may be written as

$$
\omega=\sum_{i_{1}<\cdots<i_{r}} c_{i_{1} \cdots i_{r}} \frac{d \alpha_{i_{1}}}{\alpha_{i_{1}}} \wedge \cdots \wedge \frac{d \alpha_{i_{r}}}{\alpha_{i_{r}}} .
$$

Also by Theorem $1, H^{*}\left(X_{r_{i}}\right)$ is generated by those $d \alpha_{j} / \alpha_{j}$ which have $W_{i} \subset$ $V_{j}$. Thus each nonzero term in (4.1), labelled by $i_{1}, \ldots, i_{r}$, is an element of $H^{*}\left(X_{r_{k}}\right)$ where $W_{k}$ is the codimension $r$ subspace defined by the intersection of the $r$ hyperplanes $V_{i_{1}}, \ldots, V_{i_{r}}$. This provides a sum decomposition

$$
H^{r}(X)=\sum_{i=1}^{N} H^{r}\left(X_{r_{i}}\right)
$$

Theorem 3. With the $X_{r_{i}}$ defined as above, $H^{r}(X)=\bigoplus_{i=1}^{N} H^{r}\left(X_{r_{i}}\right)$, i.e., the decomposition is a direct sum.

Proof. Suppose that in $H^{r}(X)$ we have a relation

$$
\sum_{i_{1}<\cdots<i_{r}} c_{i_{1} \cdots i_{r}} \frac{d \alpha_{i_{1}}}{\alpha_{i_{1}}} \wedge \cdots \wedge \frac{d \alpha_{i_{r}}}{\alpha_{i_{r}}}=0 .
$$

For any given codimension $r$ subspace $W$ occurring in the list $W_{1}, \ldots, W_{N}$ we select all those terms in (4.2) such that $\alpha_{i_{j}}$ vanishes on $W$ for $j=1, \ldots, r$, i.e., such that $V_{i_{1}} \cap \cdots \cap V_{i_{r}}=W$. We will show that the sum of these terms alone is zero (for each choice of $W$ ), for this is equivalent to the claimed directness of the sum decomposition. 
By relabelling we may assume, without loss of generality, that $\alpha_{1}, \ldots, \alpha_{k}$ are the linear functions from among $\alpha_{1}, \ldots, \alpha_{m}$ which vanish on $W$, and that $\alpha_{1}, \ldots, \alpha_{r}$ are linearly independent. We extend this subset to a basis $\alpha_{1}, \ldots, \alpha_{r}, \beta_{1}, \ldots, \beta_{s}$ of $V$. Note that $\alpha_{r+1}, \ldots, \alpha_{k}$ are linear combinations of $\alpha_{1}, \ldots, \alpha_{r}$ while $\alpha_{k+1}, \ldots, \alpha_{m}$ must have some nonzero coefficient of $\beta_{1}, \ldots, \beta_{s}$ in their expansion with respect to this basis. In particular, as a polynomial in $\beta_{1}, \ldots, \beta_{s}$ (with coefficients which are polynomials in $\alpha_{1}, \ldots, \alpha_{r}$ ) the product $\alpha_{k+1} \cdots \alpha_{m}$ has degree $m-k$, and each monomial $\mu$ of this degree has a constant coefficient $A_{\mu}$ (at least one of which is nonzero).

Multiplying (4.2) by the product $\alpha_{1} \cdots \alpha_{m}$ we obtain

$$
\sum_{i_{1}<\cdots<i_{r}} c_{i_{1} \cdots i_{r}} d \alpha_{i_{1}} \wedge \cdots \wedge d \alpha_{i_{r}} \frac{\alpha_{1} \cdots \alpha_{m}}{\alpha_{i_{1}} \cdots \alpha_{i_{r}}}=0 .
$$

We regard (4.3) as a polynomial in $\beta_{1}, \ldots, \beta_{s}$ with coefficients in the tensor product of $\Lambda^{r}(V)$ with polynomials in $\alpha_{1}, \ldots, \alpha_{r}$. Note that any term with $\left(i_{1}, \ldots, i_{r}\right) \subset(1, \ldots, k)$ has a factor $\alpha_{k+1} \cdots \alpha_{m}$ and has

$$
A_{\mu} c_{i_{1} \cdots i_{r}} \frac{d \alpha_{i_{1}} \wedge \cdots \wedge d \alpha_{i_{r}}}{\alpha_{i_{1}} \cdots \alpha_{i_{r}}} \alpha_{1} \cdots \alpha_{k}
$$

as the coefficient of each monomial $\mu$ in $\beta_{1}, \ldots, \beta_{s}$ of degree $m-k$. All other terms have lower degree in $\beta_{1}, \ldots, \beta_{s}$. Hence each monomial $\mu$ in $\beta_{1}, \ldots, \beta_{s}$ of degree $m-k$ which occurs in the expression (4.3) must have coefficient

$$
A_{\mu} \alpha_{1} \cdots \alpha_{k} \sum_{\left(i_{1}, \ldots, i_{r}\right) \subset(1, \ldots, k)} c_{i_{1} \cdots i_{r}} \frac{d \alpha_{i_{1}} \wedge \cdots \wedge d \alpha_{i_{r}}}{\alpha_{i_{1}} \cdots \alpha_{i_{r}}}
$$

which must itself be zero. This proves the result.

\section{ARBITRARY COEFFICIENTS}

If $\mathscr{F}$ is any sheaf on $X$ one defines $\Gamma_{M}(X, \mathscr{F})$ to be the space of sections of $\mathscr{F}$ supported in $M$ and the local cohomology along $M$ to be the right derived functors of $\Gamma_{M}(X,-)$ (see e.g. [3]). The long exact sequence and Excision Theorem follow immediately from this definition. Our explicit form of the Thom isomorphism depends only on the Künneth formula and cohomology of $\mathbb{C}^{*}$, and so it holds for any constant sheaf of rings $R$. Theorem 1 requires only these results and so remains true at this level of generality. In Theorem 2 we used the Euler operator. However, the Euler operator is defined for any exterior algebra as the derivation of degree -1 which takes the value 1 on the generators and all of the arguments of Theorem 2 remain valid in this context. The relations described in Theorem 2 all have coefficients 1 or -1 , which are units in any ring, so that the relations can be "eliminated" to give a set of free generators for $H^{*}(X, R)$. In summary we have

Theorem 4. Let $X$ be a complement of $m$ hyperplanes. For any ring of coefficients $R$ the cohomology ring $H^{*}(X, R)$ is the quotient of the exterior $R$-algebra 
on $m$ generators $e_{1}, \ldots, e_{m}$ by the ideal generated by $\Upsilon \neg\left(e_{i_{1}} \wedge \cdots \wedge e_{i_{k}}\right)$ for those subsets of defining equations $\alpha_{i_{1}}, \ldots, \alpha_{i_{k}}$ of the hyperplanes which are dependent. In particular $H^{*}(X, R)$ is freely and finitely generated over $R$ and $H^{*}(X, \mathbb{Z})$ is torsion free.

\section{REFERENCES}

1. R. Bott and L. W. Tu, Differential forms in algebraic topology, Graduate Texts in Math. 82, Springer-Verlag, New York, 1982.

2. E. Brieskorn, Sur les groupes de tresses [d'après V. I. Arnold], Sem. Bourbaki, $24^{i e ̀ m e}$ année (1971/1972), Exp. No. 401, Lecture Notes in Math., vol. 317, Springer, Berlin, 1973, pp. 21-44.

3. B. Iversen, Cohomology of sheaves, Springer-Verlag Universitext, Berlin, 1985.

4. P. Orlik, Introduction to arrangements, Amer. Math. Soc. Regional Conf. Ser. in Math., no. 72, Amer. Math. Soc., Providence, RI, 1988.

5. P. Orlik and L. Solomon, Combinatorics and topology of complements of hyperplanes, Invent. Math. 56 (1980), 167-189.

6. P. Orlik, L. Solomon and H. Terao, Arrangements of hyperplanes and differential forms, Contemp. Math., vol. 34, Amer. Math. Soc., Providence, RI, 1984, pp. 29-65.

Department of Mathematics, Victoria University, GPO Box 2476, Melbourne 3001 VIC, Australia

School of Information Sciences and Technology, The Flinders University of South australia, GPO Box 2100, Adelaide 5001 SA, Australia 